Copyright (@ 2009 Institute of Electrical and electronics Engineers, Inc.

All Rights reserved.

Personal use of this material, including one hard copy reproduction, is permitted.

Permission to reprint, republish and/or distribute this material in whole or in part for any other purposes must be obtained from the IEEE.

For information on obtaining permission, send an e-mail message to stds-igr@ieee.org.

By choosing to view this document, you agree to all provisions of the copyright laws protecting it.

Individual documents posted on this site may carry slightly different copyright restrictions.

For specific document information, check the copyright notice at the beginning of each document. 


\title{
State Feedback Controller Design of Networked Control Systems
}

\author{
Dong Yue, Qing-Long Han, and Chen Peng
}

\begin{abstract}
This paper is concerned with the controller design of networked control systems (NCSs). A new model of the NCSs is provided under consideration of both the network-induced delay and the data packet dropout in the transmission. In terms of the given model, a controller design method is proposed based on a delay-dependent approach. The feedback gain of a memoryless controller and the maximum allowable value of the network-induced delay can be derived by solving a set of linear matrix inequalities. Two examples are given to show the effectiveness of our method.
\end{abstract}

Index Terms-Lyapunov functional, networked control system (NCS), network-induced delay, stability.

\section{INTRODUCTION}

$\mathbf{R}$ ECENTLY, much attention has been paid to the study of stability analysis and control design of networked control systems (NCSs) [2], [11], [15] due to their low cost, reduced weight and power requirements, simple installation and maintenance, and high reliability. In an NCS, one of the important issues to treat is the effect of the network-induced delay on the system performance. For NCSs with different scheduling protocols, the network-induced delay may be constant, time-varying, or even random variable [15].

A basic problem in an NCS is the stability of the system. Based on a hybrid system technique or stability region method, stability of the NCSs has been investigated in [15] under an assumption that the network-induced delay is less than the sampling period. Moreover, most of the results in [15] were derived for the case of constant delay. Under the assumption that only data messages from a sensor are transmitted through a network and the considered NCS can be approximated by a continuous-time model, a perturbation method of stability analysis of the NCSs was given in [13]. A model-based method for stability

Manuscript received December 25, 2003; revised March 17, 2004. The work of D. Yue and Q.-L. Han was supported in part by Central Queensland University for the 2004 Research Advancement Awards Scheme Project "Analysis and Synthesis of Networked Control Systems." The work of D. Yue was also supported in part by the Teaching and Research Award Program for Outstanding Young Teachers at Nanjing Normal University and the Key Scientific Research Foundation by the Ministry of Education of China (03045). This paper was recommended by Associate Editor X. Wang.

D. Yue is with the Department of Control Science and Engineering, Nanjing Normal university, Nanjing, Jiangsu 210042, China, and with the Faculty of Informatics and Communication, Central Queensland University, Rockhampton, QLD 4702, Australia (e-mail: medongy@ pine.njnu.edu.cn).

C. Peng is with the Department of Control Science and Engineering, Nanjing Normal University, Nanjing, Jiangsu 210042, China.

Q.-L. Han is with the Faculty of Informatics and Communication, Central Queensland University, Rockhampton, QLD 4702, Australia (e-mail: q.han@cqu.edu.au).

Digital Object Identifier 10.1109/TCSII.2004.836043 analysis of the NCSs was recently proposed in [9], which however can only be used to treat the systems with sensor-to-controller delay case. The sampling time scheduling method was first proposed by Hong [4], which was used to appropriately select a sufficiently long sampling period for a discrete-time network-based system such that network-induced delays do not influence the control performance and the system can remain stable. However, it is necessary that the control delay in a control loop is less than the sampling period of the loop. In addition, the technique in [4] can only be used in the one-dimensional case. Park et al. [12] and Kim et al. [6] extended the concept of maximum allowable bound in [4] to develop another algorithm for the multidimensional cases. However, only the time-invariant delay case was considered in [6] and [12]. On the other hand, in all of the above works, for the stability analysis, the control law used was designed in advance without considering the presence of the network. No controller synthesis method was given for the NCSs.

The focus has been on the controller design of the NCSs in recent years, and several controller design methods have been proposed under different assumptions on the considered NCS. The design methods of stochastic optimal controller were proposed based on a discrete-time model for the cases when the network-induced delay is shorter [11] or larger [5] than the sampling period. The drawback of these methods is a large requirement of controller memory to store a large amount of past information from the initial point [2]. Moreover, to implement the controller, the information of all past delays must be known a priori and the dynamic model of the plant has to be very precise. In [1], a predictor-based delay compensation method was employed to design the memory feedback controller. However, in these methods, the effect of controller-to-actuator delays was neglected. It should be noted that the discrete-time model was used in the above references, which may lose the information on the intersample behavior when discretizing the continuous-time plant. Moreover, no method was given in the above references of how to estimate the maximum allowable value of the network-induced delay that guarantees the stabilizability of the NCSs.

In this paper, we will be concerned with the controller design for the NCSs. Under consideration of network-induced delay and data packet dropout phenomenon in the transmission, a new model of the NCS is presented, in which the sensor is clockdriven, the controller and actuator are event driven, and the data are transmitted with a single packet. In our method, the discretization of the system model and the assumption that the controller dynamics are continuous are not needed for controller design. In contrast with the controller design method based on 
discrete-time models, our method is in the continuous-time domain, i.e., the intersample behavior is taken into account. Moreover, the network-induced delay considered in this paper is composed of the sensor-to-controller delay and the controller-to-actuator delay as well as the computation delay and can be slowly or quickly time-varying. Then, a controller design method is proposed based on a delay-dependent approach. From the derived criteria, the memoryless controller can be designed and the maximum allowable value of the network-induced delay can be determined by solving a set of linear matrix inequalities (LMIs). Furthermore, the relation between the lower bound on the transmission rate and the sampling period and the network-induced delay can also be determined by using the criteria. Two examples are finally given to show the effectiveness of our method.

\section{MODELING OF NeTwORKED CONTROL SYSTEMS}

In an NCS, the plant is a continuous system, which can be described as

$$
\dot{x}(t)=A x(t)+B u(t)
$$

where $x(t) \in R^{n}$ and $u(t) \in R^{m}$ are the state vector and control input vector, respectively, and $A$ and $B$ are two constant matrices.

Suppose that the sensor is chosen clock-driven, the controller and actuator are chosen event driven, and the data are transmitted with a single packet. Then, the real input $u(t)$ realized through a zeroth-order hold in (1) is a piecewise constant function. Furthermore, if we consider the effect of the network-induced delay and network packet dropout on the NCSs, the real control system can be modeled as

$$
\begin{aligned}
\dot{x}(t) & =A x(t)+B u(t), \quad t \in\left[i_{k} h+\tau_{k}, i_{k+1} h+\tau_{k+1}\right) \\
u\left(t^{+}\right) & =K x\left(t-\tau_{k}\right), \quad t \in\left\{i_{k} h+\tau_{k}, k=1,2, \ldots\right\}
\end{aligned}
$$

where $h$ is the sampling period, $i_{k}, k=1,2,3, \ldots$, are some integers and $\left\{i_{1}, i_{2}, i_{3}, \ldots\right\} \subset\{0,1,2,3, \ldots\} . \tau_{k}$ is the time delay, which denotes the time from the instant $i_{k} h$ when sensor nodes sample sensor data from a plant to the instant when actuators transfer data to the plant. Obviously, $\cup_{k=1}^{\infty}\left[i_{k} h+\tau_{k}, i_{k+1} h+\right.$ $\left.\tau_{k+1}\right)=\left[t_{0}, \infty\right), t_{0} \geq 0$. In this paper, we assume that $u(t)=0$ before the first control signal reaches the plant and a constant $\eta>0$ exists such that $\left(i_{k+1}-i_{k}\right) h+\tau_{k+1} \leq \eta, k=1,2, \ldots$.

Remark 1: In (2), $\left\{i_{1}, i_{2}, i_{3}, \ldots\right\}$ is a subset of $\{0,1,2,3, \ldots\}$. Moreover, it is not required that $i_{k+1}>i_{k}$. When $\left\{i_{1}, i_{2}, i_{3}, \ldots\right\}=\{0,1,2,3, \ldots\}$, it means that no packet dropout occurs in the transmission. If $i_{k+1}=i_{k}+1$, it implies that $h+\tau_{k+1}>\tau_{k}$, which includes $\tau_{k}=\tau_{0}$ and $\tau_{k}<h$ as the special cases. Therefore, (2) can be viewed as a general form of the NCS model, where the effect of the network-induced delay and data packet dropout are simultaneously considered.

\section{Controller Design}

In this section, we assume that the full state variables are available for measurements. Since the actuator is event driven, therefore, the system (2) can be rewritten as the following equivalent form:

$$
\begin{aligned}
\dot{x}(t) & =A x(t)+B K x\left(i_{k} h\right), \\
t & \in\left[i_{k} h+\tau_{k}, i_{k+1} h+\tau_{k+1}\right), \quad k=1,2, \ldots \\
x(t) & =x\left(t_{0}-\eta\right) e^{A\left(t-t_{0}+\eta\right)} \triangleq \phi(t), \\
t & \in\left[t_{0}-\eta, t_{0}\right]
\end{aligned}
$$

where $\phi(t)$ can be viewed as the initial condition function of the system. It is easy to see that the solutions of (3) and (4) are continuous on $\left[t_{0}, \infty\right)$.

Next, we present a controller design method for system (3) and (4) based on a Lyapunov functional method.

Definition 1: The system (3) and (4) with a feedback gain $K$ is said to be exponentially asymptotically stable if there exist constants $\alpha>0$ and $\beta>0$ such that $\|x(t)\| \leq \alpha \sup _{t_{0}-\eta \leq s<t_{0}}\|\phi(s)\| e^{-\beta t}, t \geq t_{0}$. Also, if there exists a matrix $K$ such that the system (3) and (4) with the feedback gain $K$ is exponentially asymptotically stable, then the system (2) is said to be stabilizable.

Theorem 1: For given scalars $\eta$ and $\rho_{j}>0(j=2,3)$, if there exist matrices $\tilde{P}>0, \tilde{T}>0$, a nonsingular $X$ and matrices $Y$ and $\tilde{N}_{i}(i=1,2,3)$ of appropriate dimensions such that (5) and (6), shown at the bottom of the page, are true, then the system (3) and (4) with $K=Y X^{-T}$, and consequently, (2), is exponentially asymptotically stable.

Proof: Construct a Lyapunov functional as

$$
V(t)=x^{T}(t) P x(t)+\int_{t-\eta}^{t} \int_{s}^{t} \dot{x}^{T}(v) T \dot{x}(v) d v d s
$$

where $P>0$ and $T>0$.

Taking the time derivative of $V(t)$ for $t \in$ $\left[i_{k} h+\tau_{k}, i_{k+1} h+\tau_{k+1}\right)$ and using (3) and formula $x(t)-x\left(i_{k} h\right)-\int_{i_{k} h}^{t} \dot{x}(s) d s=0$ yields

$$
\begin{aligned}
\dot{V}(t)= & 2 x^{T}(t) P \dot{x}(t) \\
& +2\left[x^{T}(t) N_{1}+x^{T}\left(i_{k} h\right) N_{2}+\dot{x}^{T}(t) N_{3}\right] \\
& \cdot\left[x(t)-x\left(i_{k} h\right)-\int_{i_{k} h}^{t} \dot{x}(s) d s\right] \\
& +2\left[x^{T}(t) M_{1}+x^{T}\left(i_{k} h\right) M_{2}+\dot{x}^{T}(t) M_{3}\right] \\
& \cdot\left[-A x(t)-B K x\left(i_{k} h\right)+\dot{x}(t)\right] \\
& +\eta \dot{x}^{T}(t) T \dot{x}(t)-\int_{t-\eta}^{t} \dot{x}^{T}(s) T \dot{x}(s) d s
\end{aligned}
$$

where $N_{i}$ and $M_{i}(i=1,2,3)$ are arbitrary matrices of appropriate dimensions and $\dot{V}(t)=$ $\limsup _{\delta \rightarrow 0^{+}}(1 / \delta)[V(t+\delta)-V(t)][3]$.

$$
\begin{aligned}
& {\left[\begin{array}{cccc}
\tilde{N}_{1}+\tilde{N}_{1}^{T}-A X^{T}-X A^{T} & \tilde{N}_{2}^{T}-\tilde{N}_{1}-\rho_{2} X A^{T}-B Y & \tilde{N}_{3}^{T}-\rho_{3} X A^{T}+X^{T}+\tilde{P} & \eta \tilde{N}_{1} \\
* & -\tilde{N}_{2}-\tilde{N}_{2}^{T}-\rho_{2} B Y-\rho_{2} Y^{T} B^{T} & -N_{3}^{T}+\rho_{2} X^{T}-\rho_{3} Y^{T} B^{T} & \eta \tilde{N}_{2} \\
* & * & \rho_{3} X+\rho_{3} X^{T}+\eta \tilde{T} & \eta \tilde{N}_{3} \\
* & * & * & -\eta \tilde{T}
\end{array}\right]<0} \\
& \left(i_{k+1}-i_{k}\right) h+\tau_{k+1} \leq \eta, \quad k=1,2, \ldots
\end{aligned}
$$


From (6), it can be seen that, when $t \in$ $\left[i_{k} h+\tau_{k}, i_{k+1} h+\tau_{k+1}\right)$

$$
-\int_{t-\eta}^{t} \dot{x}^{T}(s) T \dot{x}(s) d s \leq-\int_{i_{k} h}^{t} \dot{x}^{T}(s) T \dot{x}(s) d s
$$

and

$$
\begin{array}{r}
-2\left[x^{T}(t) N_{1}+x^{T}\left(i_{k} h\right) N_{2}+\dot{x}^{T}(t) N_{3}\right] \int_{i_{k} h}^{t} \dot{x}(s) d s \\
\leq \eta e^{T}(t) N T^{-1} N^{T} e(t)+\int_{i_{k} h}^{t} \dot{x}^{T}(s) T \dot{x}(s) d s
\end{array}
$$

where $e^{T}(t)=\left[\begin{array}{lll}x^{T}(t) & x^{T}\left(i_{k} h\right) & \dot{x}^{T}(t)\end{array}\right]$ and $N^{T}=$ $\left[\begin{array}{lll}N_{1}^{T} & N_{2}^{T} & N_{3}^{T}\end{array}\right]$.

Combining (8)-(10), we obtain

$$
\begin{gathered}
\dot{V}(t) \leq\left[\begin{array}{lll}
x^{T}(t) & x^{T}\left(i_{k} h\right) & \dot{x}^{T}(t)
\end{array}\right] \Omega\left[\begin{array}{c}
x(t) \\
x\left(i_{k} h\right) \\
\dot{x}(t)
\end{array}\right], \\
t \in\left[\begin{array}{ll}
\left.i_{k} h+\tau_{k}, i_{k+1} h+\tau_{k+1}\right)
\end{array}\right.
\end{gathered}
$$

where $\Omega$ is given in the first equation shown at the bottom of the page.

By Schur complements, $\Omega<0$ is equivalent to (12), shown at the bottom of the page.

Define $M=M_{1}, M_{2}=\rho_{2} M$, and $M_{3}=\rho_{3} M$, where $\rho_{3} \neq 0$. Thus, $\Omega<0$ implies that $M$ is nonsingular. Furthermore, defining $X=M^{-1}, Y=K X^{T}, \tilde{P}=X P X^{T}, \tilde{N}_{i}=$ $X N_{i} X^{T}, \tilde{M}_{i}=X M_{i} X^{T}$, and $\tilde{T}=X T X^{T}$ and then pre, postmultiplying both sides of (12) with $\operatorname{diag}\left(\begin{array}{llll}X & X & X & X) \text { and }\end{array}\right.$ its transpose, respectively, we can show that the solvability of (5) implies that of (12). From (11), we obtain

$$
\begin{aligned}
\dot{V}(t) & \leq-\lambda\|x(t)\|^{2}-\lambda\|\dot{x}(t)\|^{2}, \\
t & \in\left[i_{k} h+\tau_{k}, i_{k+1} h+\tau_{k+1}\right), \quad k=1,2, \ldots .
\end{aligned}
$$

where $\lambda=\lambda_{\min }(-\Omega)$.

Defining a new function $W(t)=e^{\varepsilon t} V(t)$ and then using the similar analysis method in [8], it can be seen that there exist a small enough constant $\varepsilon>0$ and a constant $\rho>0$ such that

$$
V(t) \leq \rho \sup _{t_{0}-\eta \leq s \leq t_{0}}\|\phi(s)\|^{2} e^{-\varepsilon t}, \quad t \geq t_{0}
$$

which can further imply from (7) that

$$
\|x(t)\| \leq \sqrt{\lambda_{\min }^{-1}(P) \rho} \sup _{t_{0}-\eta \leq s \leq t_{0}}\|\phi(s)\| e^{-\varepsilon t / 2}, \quad t \geq t_{0} .
$$

Then, by the Definition 1, we can complete the proof.
Remark 2: Since $x\left(i_{k} h\right)=x\left[t-\left(t-i_{k} h\right)\right]$, define $\tau(t)=$ $t-i_{k} h, t \in\left[i_{k} h+\tau_{k}, i_{k+1} h+\tau_{k+1}\right), k=1,2,3, \ldots$, then (3) becomes

$$
\begin{aligned}
& \dot{x}(t)=A x(t)+B K x(t-\tau(t)) \\
& x(t)=\phi(t), \quad t \in\left[t_{0}-\eta, t_{0}\right] .
\end{aligned}
$$

From the definition of $\tau(t)$, it can be seen that $\tau(t)$ is discontinuous at the points $i_{k} h+\tau_{k}, k=1,2,3, \ldots$ In every internal $\left[i_{k} h+\tau_{k}, i_{k+1} h+\tau_{k+1}\right), \tau_{k} \leq \tau(t) \leq\left(i_{k+1}-i_{k}\right) h+\tau_{k+1}$. If $i_{k+1}-i_{k}$ and $\tau_{k}$ are upper bounded, then $\tau(t)$ is a bounded piecewise continuous function. Therefore, the NCS system (3) and (4) is equivalent to a linear system with a bounded timevarying delay. For the case when $\tau(t)$ is time-invariant, i.e., $\tau(t)=\tau_{0}$, where $\tau_{0}$ is a constant, the controller design problem of (15) has been investigated in [6], [10], [12], and [14] based on a reduction method or a first-order transformation. However, the results there cannot be applicable to the system (15) since $\tau(t)$ here is a time-varying and discontinuous function.

Remark 3: $i_{k+1}<i_{k}$ means that the new data packet may reach the plant before the old one. However, from Theorem 1, it can be shown that this phenomena may lead to a conservative result. In fact, suppose $i_{k+1}<i_{k}<i_{k+2}$ and $\eta$ satisfying (5) is solved; from (6), it is required that $\left(i_{k+2}-i_{k+1}\right) h+\tau_{k+2} \leq \eta$. Since $i_{k+2}-i_{k}<i_{k+2}-i_{k+1}$, thus, for the same $\tau_{k+2}$, discarding the old data packet containing $x\left(i_{k+1} h\right)$ may result in a less conservative result. On the other hand, suitably discarding the old data packet can save network bandwidth and thus reduce the networked-induced delay $\tau_{k}(k=1,2, \ldots)$, which in turn makes the system be able to tolerate a larger amount of data packet loss. Therefore, it is necessary to find an appropriate network scheduling method that can discard the old untransmitted message when the new packet is transmitted. In the following discussion, we assume that $i_{k+1}>i_{k}, k=1,2,3, \ldots$.

For the following use, we give two definitions.

Definition 2: The maximum transmission index $m$ is defined as the supremum of $i_{k+1}-i_{k}, k=1,2, \ldots$, satisfying (6).

Remark 4: Obviously, $m$ is an integer.

Definition 3: The lower bound on transmission rate $\gamma$ is defined as $1 / m$, where $m$ is as defined in Definition 2.

Remark 5: $\gamma$ in Definition 3 is a parameter, which denotes the degree of the data packet dropout that the feedback control system can tolerate. Different from [15], the data packet dropout considered in this paper may occur in the network not only from the plant to control but also from the controller to actuator.

$$
\Omega=\left[\begin{array}{ccc}
N_{1}+N_{1}^{T}-M_{1} A-A^{T} M_{1}^{T} & N_{2}^{T}-N_{1}-A^{T} M_{2}^{T}-M_{1} B K & N_{3}^{T}-A^{T} M_{3}^{T}+M_{1}+P \\
* & -N_{2}-N_{2}^{T}-M_{2} B K-K^{T} B^{T} M_{2}^{T} & -N_{3}^{T}+M_{2}-K^{T} B^{T} M_{3}^{T} \\
* & * & M_{3}+M_{3}^{T}+\eta T
\end{array}\right]+\eta N T^{-1} N^{T}
$$

$$
\left[\begin{array}{cccc}
N_{1}+N_{1}^{T}-M_{1} A-A^{T} M_{1}^{T} & N_{2}^{T}-N_{1}-A^{T} M_{2}^{T}-M_{1} B K & N_{3}^{T}-A^{T} M_{3}^{T}+M_{1}+P & \eta N_{1} \\
* & -N_{2}-N_{2}^{T}-M_{2} B K-K^{T} B^{T} M_{2}^{T} & -N_{3}^{T}+M_{2}-K^{T} B^{T} M_{3}^{T} & \eta N_{2} \\
* & * & M_{3}+M_{3}^{T}+\eta T & \eta N_{3} \\
* & * & * & -\eta T
\end{array}\right]
$$


Remark 6: When $\eta$ satisfying (5) is found and $\tau_{k}$ is known to be less than a constant $\tau_{0}$, one can show that

$$
\left(i_{k+1}-i_{k}\right) h \leq \eta-\tau_{0}
$$

implies (6). If $m_{0}$ is defined as the supremum of $i_{k+1}-i_{k}$, $k=1,2, \ldots$, satisfying (16), it is easy to see that $m \geq m_{0}$. Therefore, the lower bound on transmission rate $\gamma$ is a number less than $1 / m_{0}$. From (16), it can be shown that fast sampling, i.e., decreasing $h$, can allow for a larger $m_{0}$, which in turn leads to reducing the lower bound on transmission rate $\gamma$.

Remark 7: If $i_{k}=k$, namely, no data packet dropout occurs in the transmission, (6) becomes $h+\tau_{k+1} \leq \eta$. Furthermore, if $\tau_{k}=\tau_{0}$ is a constant, which may be implemented by using static scheduling network protocols, such as a token ring or a token bus, we obtain $h+\tau_{0} \leq \eta$. From this relation, it can be concluded that faster sampling can allow for a larger networked-induced delay. Moreover, different from the method in [15, Example 2 and Corollary 4], it is not required that $\tau_{0}<h$. For the conventional computer control systems, the data packet dropout due to the bandwidth limit does not exist and the networked-induced delay can be ignored, therefore, $\tau_{k}=0$ and $i_{k}=k$. In this case, (6) becomes $h \leq \eta$. Therefore, Theorem 1 provides a criterion that can determine the maximum allowable size of the sampling period.

Remark 8: From the proof of Theorem 1, to derive the condition (5), $M_{2}$ and $M_{3}$ are set as $\rho_{2} M$ and $\rho_{3} M$, which results in that (12) and (5) are not equivalent. Therefore, it can be expected that the controller designed by the condition (5) can guarantee the asymptotic stability of the system with a larger bound on $\eta$ than the one derived based on (5).

From the proof of Theorem 1, we can conclude the following corollary.

Corollary 2: For given a scalar $\eta$ and a matrix $K$, if there exist matrices $P>0, T>0, N_{i}$, and $M_{i}(i=1,2,3)$ of appropriate dimension such that (17) and (18), shown at the bottom of the page, are true, then the system (3) and (4), and, consequently, (2) with the feedback gain $K$, is exponentially asymptotically stable.

Step 1) Set $\rho_{2}=\delta_{2}$ and $\rho_{3}=\delta_{3}$ and choose a small constant $\varepsilon$ and a constant $\beta$, where $\beta>\max \left\{\delta_{2}, \delta_{3}\right\}$. Find the maximum allowable value of $\eta_{\max }$ satisfying (5) and solve the feedback gain $K=Y X^{-T}$. Set $\eta_{0}=\eta_{\max }$ and $K_{0}=K$.

Step 2) Set $\rho_{3}=\rho_{3}+\varepsilon$. If $\rho_{3}>\beta$, go to Step 3. Otherwise, find the maximum allowable value of $\eta_{\max }$ satisfying (5) and solve the feedback gain $K=Y X^{-T}$. If $\eta_{\max }>\eta_{0}$, then set $\eta_{0}=\eta_{\max }$ and $K_{0}=K$. Repeat Step 2.
Step 3) Set $\rho_{2}=\rho_{2}+\varepsilon$. If $\rho_{2}>\beta$, go to Step 4. Otherwise, set $\rho_{3}=\delta_{3}$ and go to Step 2 .

Step 4) Substituting $K=K_{0}$ in Step 2 into (17) and find the maximum allowable value of $\eta_{\max }$.

Step 5) Output $\eta_{\max }$ and $K$ and then Stop.

Remark 9: It is easy to see that $\eta_{\max }$ solved based on the above algorithm is larger than the one directly obtained from Theorem 1.

\section{NUMERICAL EXAMPLE}

Example 1: Consider a system [15, Example 1]

$$
\dot{x}(t)=\left[\begin{array}{cc}
0 & 1 \\
0 & -0.1
\end{array}\right] x(t)+\left[\begin{array}{c}
0 \\
0.1
\end{array}\right] u(t) .
$$

The nonnetworked controller is designed as $u(t)=K x(t)$, where the feedback gain $K=\left[\begin{array}{ll}-3.75 & -11.5\end{array}\right]$. From [12] and [15], the maximum allowable transfer interval (MATI), also called MADB [6], that guarantees the stability of the system (19) controlled over a network is $4.5 \times 10^{-4}$ and 0.0538 , respectively. By using [6, Theorem I], it can be computed that the MATI is 0.7805 . As pointed in [6], determination of the MATI for an NCS is important for us to choose a suitable sampling period. If the sampling period exceeds the given MATI, then the stability of the overall system could not be guaranteed [6]. It should be noted that the maximum stable constant sampling period for this feedback system by using a stability region technique [15] is 1.7, which shows that the above given results may be conservative. Applying Corollary 1, it shows that the MATI is 0.8695 even for the fast time-varying delay case. Obviously, our result is less conservative than the ones based on the methods in [6], [12], and [15].

In [6], [12], and [15], it was assumed that the controller dynamics in (19) is continuous and/or the communication medium is error-free [13]. However, the real controller in an NCS is a piecewise-constant function and the network-induced delay is often time-varying [2], therefore, the methods in [6], [12], and [15] cannot be applicable to the system (19) when the controller is computer-based and the control input signal reaches the plant through the network. In this case, (19) can be expressed as (2) with $A=\left[\begin{array}{cc}0 & 1 \\ 0 & -0.1\end{array}\right]$ and $B=\left[\begin{array}{c}0 \\ 0.1\end{array}\right]$, which is denoted as (19). It has been computed that $\eta_{\max }$ is 0.8695 when $K=\left[\begin{array}{ll}-3.75 & -11.5\end{array}\right]$. From the relation (18), it can be seen that, when the sampling period $h$ is sufficiently small, the maximum allowable value of the time delay $\tau_{k}$ will approach 0.8695 . When the sampling period $h$ is chosen as in [15], i.e., $h=0.3 \mathrm{~s}$, and furthermore the network-induced delay is bounded by 0.1 , that is, $\tau_{k} \leq 0.1, k=1,2, \ldots$, then it can be computed that the lower bound on transmission rate $\gamma$ is less than $1 / 2$.

$$
\begin{aligned}
& {\left[\begin{array}{cccc}
N_{1}+N_{1}^{T}-M_{1} A-A^{T} M_{1}^{T} & N_{2}^{T}-N_{1}-A^{T} M_{2}^{T}-M_{1} B K & N_{3}^{T}-A^{T} M_{3}^{T}+M_{1}+P & \eta N_{1} \\
* & -N_{2}-N_{2}^{T}-M_{2} B K-K^{T} B^{T} M_{2}^{T} & -N_{3}^{T}+M_{2}-K^{T} B^{T} M_{3}^{T} & \eta N_{2} \\
* & * & M_{3}+M_{3}^{T}+\eta T & \eta N_{3} \\
* & * & * & -\eta T
\end{array}\right]<0} \\
& \left(i_{k+1}-i_{k}\right) h+\tau_{k+1} \leq \eta, \\
& k=1,2, \ldots
\end{aligned}
$$


Among the above references, no controller synthesis method was given for this example when considering the presence of a network. Next, we consider this problem and derive the maximum allowable value of $\eta_{\max }$ that guarantees the stabilizability of the system (19). For this purpose, we apply Algorithm 1. Applying Algorithm 1 with $\rho_{2}=0.2$ and $\rho_{3}=20$, it has been found that the maximum allowable value of $\eta_{\max }$ is 402 and the corresponding feedback gain is $K=\left[\begin{array}{ll}-0.0025 & -0.0118\end{array}\right]$. In other words, as long as $\left(i_{k+1}-i_{k}\right) h+\tau_{k+1} \leq 402, k=1,2, \ldots$, (19) with $K=\left[\begin{array}{ll}-0.0025 & -0.0118\end{array}\right]$ is asymptotically stable. If $h$ is chosen as 1 and $\sup _{k}\left\{\tau_{k}, k=1,2, \ldots\right\} \leq 10$, then $m_{0}=392$. Therefore, it can be computed that the lower bound on transmission rate $\gamma$ is less than $1 / 392$.

Example 2: Consider a simplified model of the inverted system process [5]

$$
\dot{x}(t)=\left[\begin{array}{ll}
0 & 1 \\
1 & 0
\end{array}\right] x(t)+\left[\begin{array}{l}
0 \\
1
\end{array}\right] u(t) .
$$

When the controller is implemented through a network, the system (20) can be written in the form of (2). A stochastic optimal control of (20) was designed in [5]. However, only the case when the network-induced delay is less than the sampling period was considered in [5]. Suppose that the full state variables are available for measurement. Applying Algorithm 1 with $\rho_{2}=0.2$ and $\rho_{3}=20$, it has been found that the maximum allowable value of $\eta_{\max }$ that guarantees the stabilizability of the system (20) is 0.97. If $h=0.05 \mathrm{~s}$ and the data packet dropout in the transmission can be neglected, from (18), the time delay $\tau_{k} \leq 0.92=18.4 \mathrm{~h}$. That means that, if $h=0.05 \mathrm{~s}$, the designed controller can stabilize the system (20) as long as the network-induced delay is less than $18.4 \mathrm{~h}$. Furthermore, if the upper bound of the network-induced delay $\tau_{k}$ is known as 0.3 , i.e., $6 \mathrm{~h}$, then it can be computed that the lower bound on transmission rate $\gamma$ is less than $1 / 13$.

\section{CONCLUSION}

The methods of stability analysis and controller design have been proposed based on a new model of the NCS. In the given model, both the network-induced delay and the data packet dropout were simultaneously, considered and no discretization of the continuous-time plant was needed for modeling. Therefore, our analysis was in the continuous-time domain, i.e., the intersample behavior was taken into account. The stability analysis and controller design can be performed and furthermore the relation between the sampling period and the network-induced delay can also be determined by solving a set of LMIs. Combining the robustness analysis approaches proposed for the nonnetworked control systems [7], our method in this paper can be further extended to the study of stability and controller design for the NCSs when modeling errors or even nonlinearities exist in the systems. In addition, since (2) is a general model for the NCS with a direct structure or a hierarchical structure [2], the methods of analysis and controller design in this paper can be available for the real NCSs cited in many existing works [6], [15]. It should be pointed out that we only consider the case of single-packet transmission in this paper, which is available for the systems based on Ethernet. Furthermore, by emplying a FIFO queue [2] before the controller and actuator, the methods in this paper can be easily extended to deal with the controller design of the NCSs with multiple-packet transmission, such as the systems based on DeviceNet. This work will be left for our future research.

\section{ACKNOWLEDGMENT}

The authors would like to thank the Associate Editor and the anonymous reviewers for their constructive comments and suggestions to improve the quality of the paper.

\section{REFERENCES}

[1] H. Chan and U. Ozguner, "Closed-loop control of systems over a communication network with queues," Int. J. Control, vol. 62, pp. 493-510, 1995.

[2] M. Y. Chow and Y. Tipsuwan, "Network-based control systems: A tutorial,' in Proc. IECON'01: 27th Annu. Conf. IEEE Industrial Electronics Soc., 2001, pp. 1593-1602.

[3] J. Hale and S. M. V. Lunel, Introduction to Functional Differential Equations. New York: Springer-Verlag, 1993.

[4] S. H. Hong, "Scheduling algorithm of data sampling times in the integrated communication and control systems," IEEE Trans. Contr. Syst. Technol., vol. 3, pp. 225-230, June 1995.

[5] S. S. Hu and Q. X. Zhu, "Stochastic optimal control and analysis of stability of networked control systems with long delay," Automatica, vol. 39, pp. 1877-1884, 2003.

[6] D. S. Kim, Y. S. Lee, W. H. Kwon, and H. S. Park, "Maximum allowable delay bounds of networked control systems," Control Eng. Practice, vol. 11, pp. 1301-1313, 2003.

[7] M. S. Mahmoud, Robust Control and Filtering for Time-Delay Systems. New York: Marcel Dekker, 2000.

[8] X. Mao, N. Koroleva, and A. Rodkina, "Robust stability of uncertain stochastic differential delay equations," Syst. Contr. Lett., vol. 35, pp. 325-336, 1998

[9] L. A. Montestruque and P. J. Antsaklis, "On the model-based control of networked systems," Automatica, vol. 39, pp. 1837-1843, 2003.

[10] Y. S. Moon, P. Park, and W. H. Kwon, "Robust stabilization of uncertain input-delayed systems using reduction method," Automatica, vol. 37, pp. 307-312, 2001.

[11] J. Nilsson, B. Bernhardsson, and B. Wittenmark, "Stochastic analysis and control of real-time systems with random time delays," Automatica, vol. 34, pp. 57-64, 1998.

[12] H. S. Park, Y. H. Kim, D. S. Kim, and W. H. Kwon, "A scheduling method for network based control systems," IEEE Trans. Contr. Syst. Technol., vol. 10, pp. 318-330, May 2002.

[13] G. C. Walsh, O. Beldiman, and L. G. Bushnell, "Asymptotic behavior of nonlinear networked control systems," IEEE Trans. Automat. Contr., vol. 46, pp. 1093-1097, July 2001.

[14] D. Yue, "Robust stabilization of uncertain systems with unknown input delay," Automatica, vol. 40, pp. 331-336, 2004.

[15] W. Zhang, M. S. Branicky, and S. M. Phillips, "Stability of networked control systems," IEEE Control Syst. Mag., vol. 21, pp. 84-99, Feb. 2001. 